\title{
THE DEVELOPMENT OF AN OPTIMAL BONUS-MALUS SYSTEM IN A COMPETITIVE MARKET
}

\author{
BY \\ Fabio Baione, Susanna Levantesi and Massimiliano Menzietti \\ Department of Actuarial Science, University "La Sapienza", \\ Rome, Italy
}

\begin{abstract}
BMS in force show a progressive reduction of the observed average premium, which causes a financial imbalance in the system (see LEMAIRE (1995)). As a consequence, frequent premium adjustments become necessary and result in a discrepancy between the reduction defined in the policy contract and the effective discount applied to the driver. Most policyholders are not aware of this "lack of transparency". This paper deals with the problem of designing an optimal tariff structure so that the designed BMS is adequate and satisfies both transparency and financial balance conditions.
\end{abstract}

\section{KEYWORDS}

Bonus-Malus systems, Stationary distribution, Transparency, Adequacy

\section{INTRODUCTION}

The recent implementation of a European regulation on non-life insurance (the "Third Directive", No. 92/49/EEC) introduced new elements of competition in the Italian motor insurance market. The end of the fixation of premia by the State produced new interest in the development of pricing models for automobile insurance.

In the development of a tariff structure for automobile insurance, a priori classification is not fully efficient; therefore insurers also apply an a posteriori risk-classification. Generally, the a posteriori process of risk-classification is implemented by using Bonus-Malus Systems (BMS), which provide a premium reduction (bonus) for a good driver, as well as an increment (malus) for a bad one. In fact, the BMS are built up according to the following variables' change: classes number, transition rules, starting class or level of penalisation charge. 
A structural consequence of implementing a BMS is a progressive reduction of the observed average premium. This is due to an excessive concentration of policyholders in low-charged classes, and to an insufficient penalisation of the "bad" insured (see LeMAIRE (1985), (1995)). This reduction causes a financial imbalance in the system; therefore frequent premium adjustments become necessary. The system can be rebalanced by changing the transition rules, by changing the premium coefficients, or — as is usually the case - by increasing premiums in all classes by a constant percentage.

In order to determine a balanced system it is necessary to consider other components, such as claim frequency and claim severity. A variation of either variable, or both, causes a change of premium in each class.

After 1994 the Italian motor insurance industry began operating in a more competitive market. Companies were able to determine their own transition rules and premium coefficients i.e. their policies design.

In this paper we focus our attention on premium coefficients, so to determine a set of premium coefficients, which satisfy some specific evaluating conditions for a BMS. For each class, the premium coefficients are calculated so that not only the designed system is adequate but it also leads to a financial balance. As "adequacy condition" we require that the system tends over time to provide a fair price to each driver.

Constant increases in the basic premium result in a discrepancy between the premium reduction, or increment, defined in the policy contract and the effective discount to the insured. Such a discrepancy is detrimental to the transparency requirement between insurer and insured.

Since our aim is to develop an optimal tariff structure, we want to include a transparency condition in the model. We also require that the system maximises adequacy, and at the same time satisfies financial balance conditions.

The model developed is fitted to data from three countries, where penalisation rules are different. In order to compare the theoretical and the observed optimal premium scales, we measure the model's ability to improve the adequacy of any policy structure.

Assuming that each country applies the same premium scales, we compare results amongst countries and evaluate the impact of the transition rules on the system adequacy.

The paper is structured as follows: Section 2 introduces the notation and defines the BMS. Section 3 analyses BM premiums and the financial balance condition. Section 4 designs the optimal tariff structure using the adequacy and transparency conditions. Section 5 reports the results of the model in systems characterised by different transition rules. Finally, we present some concluding remarks.

\section{DEFINITION OF THE BMS}

Consider a portfolio of insured risks, closed to new entries and to exits, where drivers are insured at the same time and are homogeneous with respect to some a priori characteristics. 
Let $N$ denote the random number of claims reported by a policyholder in one year and let $Y_{h}(h=1,2, \ldots, N)$ be the corresponding random claim severities, which are i.i.d. and independent of $N$. The aggregate claim amount of the policy can be written in the form $X=\sum_{h=1}^{N} Y_{h}$.

Let us assume that the average claim amount is equal to 1 . The annual claim number of a policyholder is a Poisson distributed variable, with parameter $\lambda$, where $\lambda$ is assumed to be constant over time; $\lambda$ varies from insured to insured, and is distributed as a Gamma variable, with parameters $\alpha, \beta$. The density function of the Gamma distribution is denoted $u(\lambda)$. Hence the distribution of the aggregate claim amount is a Negative Binomial (see LEMAIRE (1995), pages 29-31).

Let us consider that all policyholders are subdivided into a finite number of BMS classes $(i=1,2, \ldots, s)$, in which all transfers of the policies within these classes are regulated by defined transition rules. The class assignment to every policyholder in a given year is uniquely determined by both the class where he belonged in the previous year and the number of claims reported during the year.

All policies are placed in the same initial class, say $\eta$, for the first year.

For each merit class we define a premium coefficient $c_{i}$ that represents the ratio of the merit class to the starting class premium.

The transition rules can be modelled in the form of transformations $T_{k}$ such that $T_{k}(i)=j$ if the policy moves from class $i$ into class $j$, when $k$ claims have been reported.

This system is a first-order Markov chain with the following transition matrix:

$$
M(\lambda)=\left(p_{i j}(\lambda)\right)=\sum_{k=0}^{\infty} p_{k}(\lambda) T_{k}
$$

where $p_{i j}(\lambda)$ is the probability that a policyholder, with annual claim frequency $\lambda$, is transferred from class $i$ into class $j$ within one year. Since the BMS is a regular Markov chain, one simple eigenvalue of the transition matrix $M(\lambda)$ is 1 . The corresponding left-eigenvector is $\bar{a}(\lambda)=\left[a_{1}(\lambda), \ldots, a_{s}(\lambda)\right]$ and defines the stationary probability distribution of a policyholder characterised by his $\lambda$ (see LOIMARANTA (1972)); $a_{i}(\lambda)$ is the limit value for the probability that the policyholder, with claim frequency $\lambda$, will be in class $i$ when the number of years tends to infinity.

For the entire portfolio of the insurer, the stationary probabilities are:

$$
a_{i}=\int_{0}^{\infty} a_{i}(\lambda) u(\lambda) d \lambda \quad i=1, \ldots, s
$$

Once the system is defined, for each year $t$ the premium level $\pi_{i}(t)$ for a driver in class $i$ is computed as a product of a basic premium ${ }^{B} P_{t}$ and an adjustment coefficient $c_{i}$; a driver in class $i$ pays a premium level equal to $\pi_{i}(t)=$ ${ }^{B} P_{t} c_{i}$. 


\section{BM PREMIUMS AND FINANCIAL BALANCE}

Let us assume that there are no expense loadings; therefore the aggregate expected claim amount is obtained by multiplying the expected value of the claim number by the average claim amount, i.e. $E(X)=E(N) E(Y)=\bar{\lambda}$.

Regarding the whole portfolio, each year the average premium is calculated by scaling the basic premium ${ }^{B} P_{t}$ by the average premium coefficient $C_{m}(t)$.

$$
C_{m}(t)=\sum_{i=1}^{s} \int_{0}^{\infty} c_{i} p_{i}(\lambda ; t) u(\lambda) d \lambda
$$

where $p_{i}(\lambda ; t)$ is the probability that a driver, with claim frequency $\lambda$, is in class $i$ after $t$ years.

In year $t$, the system becomes financially balanced if the average premium is equal to the aggregate expected amount; therefore the following holds:

$$
\bar{\lambda}=C_{m}(t){ }^{B} P_{t} \quad(t=0,1,2, \ldots .)
$$

In subsequent years the basic premium can be calculated recursively:

$$
{ }^{B} P_{t}={ }^{B} P_{t-1} \frac{C_{m}(t-1)}{C_{m}(t)}
$$

LEMAIRE (1995) highlighted that several existing BMS show "a progressive decrease of the observed average premium level, due to a concentration of policyholders in the high discount classes". The decrease involves a change in the premium level $\pi_{i}(t)$, due to an increase in the basic premium.

Such behaviour is detrimental to the transparency of the insurance activity, because good drivers are not receiving the bonus they expect from a policy contract: most of the bonus might evaporate due to an increase in the basic premium.

\section{OPTIMAL SYSTEM DEFINITION: TRANSPARENCY AND ADEQUACY}

As previously stated, it is common practice for insurance companies to take into account the financial balance condition, without considering what we call the "transparency condition".

In practice, most BMS provide a premium coefficients structure with a number of bonus classes greater than the number of malus classes.

The annual decrease in the average premium coefficient causes a change in the basic premium, in respect of the financial balance condition. As a consequence the original premium scale is adjusted by a factor $1 / C_{m}(t)$, which in turn causes some bonus classes to become malus classes. The same effect is obtained by applying the factor to premium coefficients $c_{i}(i=1,2, \ldots, s)$ rather than correcting the basic premium. 
For instance, consider a BMS characterised by two merit classes with $c_{1}=$ 0.85 and $c_{2}=1$; drivers, at starting class two, may think they enjoy a no claim discount of $15 \%$ with transition probability of - say - 0.9. Under these assumptions, the average premium coefficient becomes 0.87 , so the original premium coefficients are $c_{1}^{\prime}=0.98$ and $c_{2}^{\prime}=1.15$ and the effective no claim discount is only $2 \%$. Most policyholders are not aware of this situation. Insurers can be criticised for this "lack of transparency". As a result, the effective merit class structure penalises the insured more than the structure described in the contract.

A BMS characterised by a set of optimal merit classes should be built in such a way that the predefined reductions and penalties be guaranteed in advance to the insured. Therefore a transparency condition must be satisfied. Ideally we would introduce it by requiring that the average premium coefficient $C_{m}(t)$ is equal to 1 at each time $t$; this way, premium adjustments are no longer necessary.

The adequacy of the system is not guaranteed by considering this constraint alone; for a system to be adequate we require that it tends, over time, to provide a fairer premium.

By comparing the premium paid by each driver to his/her fair premium, we obtain the average rating error of the whole portfolio. This provides a measure of adequacy of the system, when the stationary condition is reached. Specifically, as "adequacy measure" we introduce the following expression:

$$
A m\left(c_{i}\right)=\sum_{i=1}^{s} \int_{0}^{\infty} a_{i}(\lambda)\left[c_{i} \bar{\lambda}-\lambda\right]^{2} u_{i}(\lambda) d \lambda
$$

$A m\left(c_{i}\right)$ is the total amount of squared class errors under the stationary assumption. Each single error equals the difference between the driver's claim frequency $\lambda$ and its estimate, weighted by the stationary probability of being in class $i$.

It is important to point out that the density function used here is specific to each class.

So, the claim frequency of each policyholder is initially distributed as a Gamma $(\alpha, \beta)$ with density function, $u(\lambda)$; then, once the process becomes stationary, this frequency is distributed, for each class of membership, as a Gamma $\left(\alpha_{i}, \beta_{i}\right)$ with density function denoted $u_{i}(\lambda)$.

It is not possible to use empirical evaluation to obtain the parameter estimates; however, by using the properties of the Gamma distribution, the parameters can be computed from the average claim frequency of the class $\bar{\lambda}_{i}$, and the corresponding variance, $\sigma_{i}^{2}(\lambda)$, defined by:

$$
\bar{\lambda}_{i}=\frac{\int_{0}^{\infty} \lambda a_{i}(\lambda) u(\lambda) d \lambda}{\int_{0}^{\infty} a_{i}(\lambda) u(\lambda) d \lambda}
$$




$$
\sigma_{i}^{2}(\lambda)=\frac{\int_{0}^{\infty}\left(\lambda-\bar{\lambda}_{i}\right)^{2} a_{i}(\lambda) u(\lambda) d \lambda}{\int_{0}^{\infty} a_{i}(\lambda) u(\lambda) d \lambda}
$$

We can build an optimal system for a finite set of classes using a non-decreasing set of premium coefficients $c_{i}$, that, under the transparency condition, maximises the adequacy, i.e. minimises the average rating error calculated on portfolio (5).

As it is impossible to obtain a solution which satisfies the transparency condition at each $t$, we require that the condition be satisfied only when the process has become stationary; i.e. as $t$ tends to infinity.

$$
C_{m}(\infty)=\sum_{i=1}^{s} \int_{0}^{\infty} c_{i} a_{i}(\lambda) u(\lambda) d \lambda=1
$$

During the early years of existence of the BMS, it will still be necessary to adjust premiums levels. In the long run, adjustments will become smaller and smaller, and reductions and penalisations will correspond to those specified in the policy contract.

Hence, the optimal premium coefficients can be obtained by solving the following:

$$
\begin{cases}\operatorname{Min} & \operatorname{Am}\left(c_{i}\right) \\ & C_{m}(\infty)=1 \\ & c_{i} \leq c_{i+1} \quad \text { with } i=1,2, \ldots, s-1\end{cases}
$$

The constraints $c_{i} \leq c_{i+1}(i=1,2, \ldots, s-1)$ ensure that the premium increases with the class.

\section{NUMERICAL EXAMPLE}

For a numerical application, we analyse three different BMS with seven merit classes, which are respectively adopted in Brazil, Kenya and United Kingdom $^{1}$ : same number of classes but different penalisation levels. So we can compare different transition rules in terms of resulting adequacy.

For each country we calculate three sets of premium coefficients satisfying the transparency condition at stationarity. It is necessary to use different tariff structures to compare the optimal coefficients' ability to improve the adequacy of the system.

1 The system we are referring to is typical of the BMS used in the UK (see LEMAIRE (1995), p. 152). 
- Optimal tariff

An optimal system is built by determining the premium coefficient $c_{i}^{o}$ as a solution for the constrained optimum problem expressed in (9).

- Original tariff under transparency condition

On the basis of the given premium coefficients $c_{i}^{s}$ for the seven BMS classes - say $-S$, it is possible to obtain a new sequence of coefficients, considering the transparency condition at stationarity and derived from the following ratio:

$$
c_{i}^{T}=\frac{c_{i}^{S}}{C_{m}^{S}(\infty)} \quad i=1, \ldots, 7
$$

where the average premium coefficient at stationarity $C_{m}^{S}(\infty)=\sum_{i=1}^{7} c_{i}^{S} a_{i}$ is
computed by using the original tariff structure.

- Risk classes based tariff

Assuming that all individuals in the same class are characterised by the same claim frequency, the system is built through risk classes instead of merit classes. Coefficients are given by the average claim frequency in the class at stationarity divided by the average claim frequency of the whole portfolio.

$$
c_{i}^{R}=\frac{\bar{\lambda}_{i}}{\bar{\lambda}} \quad i=1, \ldots, 7
$$

It is worth noting that the same solution is obtained in (5) by using the population density function, $u(\lambda)$, rather than $u_{i}(\lambda)$.

We use as Gamma parameters the following values ${ }^{2}: \alpha=1.96$ and $\tau=14$, corresponding to an annual frequency $\bar{\lambda}=0.14$.

The features of the three BMS are as follows.

- Premium coefficients

TABLE 1

Premium COEFFICIENTS

\begin{tabular}{lccccccc}
\hline \multirow{2}{*}{ Country } & \multicolumn{7}{c}{ Merit classes } \\
\cline { 2 - 8 } & $\mathbf{1}$ & $\mathbf{2}$ & $\mathbf{3}$ & $\mathbf{4}$ & $\mathbf{5}$ & 6 & 7 \\
\hline Kenya & 0.40 & 0.50 & 0.60 & 0.70 & 0.80 & 0.90 & 1.00 \\
UK & 0.33 & 0.40 & 0.45 & 0.55 & 0.65 & 0.75 & 1.00 \\
Brazil & 0.65 & 0.70 & 0.75 & 0.80 & 0.85 & 0.90 & 1.00 \\
\hline \hline
\end{tabular}

2 The values used derive from the whole Italian insured population in 1992. 
- Transition rules

$$
\begin{array}{ll}
\text { Kenya } & T_{k}(i)=\left\{\begin{array}{lll}
\text { if } k=0 & \max (i-1,1) \\
\text { if } k \geq 1 & 7
\end{array}\right. \\
\text { UK } & T_{k}(i)=\left\{\begin{array}{lll}
\text { if } k=0 & i=1,2, \ldots, 7 & \max (i-1,1) \\
\text { if } k=1 & \begin{cases}4 \leq i \leq 7 & i+k \\
2 \leq i \leq 3 & i+2 k \\
i=1 & i+3 k\end{cases} \\
\text { if } k=2 & \left\{\begin{array}{lll}
2 \leq i \leq 7 & i+2 k \\
i=1 & i+3 k-1
\end{array}\right. \\
\text { if } k \geq 3 & i=1,2, \ldots, 7 & 7
\end{array}\right. \\
\text { Brazil } & T_{k}(i)=\left\{\begin{array}{lll}
\text { if } k=0 & \max (i-1,1) \\
\text { if } k \geq 1 & \min (i+k, 1)
\end{array}\right.
\end{array}
$$

Applying (1), we obtain the stationary probabilities values for each merit class as reported in Table 2. Using the parameters estimated from (6) and (7) we compute the claim frequency density function $u_{i}(\lambda)$ for the specific class $i$, as reported in the appendix.

TABLE 2

STATIONARY PROBABILITIES

\begin{tabular}{lccccccc}
\hline \hline Country & $\boldsymbol{a}_{\boldsymbol{1}}$ & $\boldsymbol{a}_{\mathbf{2}}$ & $\boldsymbol{a}_{3}$ & $\boldsymbol{a}_{\mathbf{4}}$ & $\boldsymbol{a}_{5}$ & $\boldsymbol{a}_{6}$ & $\boldsymbol{a}_{7}$ \\
\hline Kenya & 0.497041 & 0.052568 & 0.061441 & 0.072438 & 0.086237 & 0.103793 & 0.126482 \\
UK & 0.641508 & 0.075324 & 0.089223 & 0.106627 & 0.046978 & 0.025048 & 0.015253 \\
Brazil & 0.828467 & 0.111779 & 0.033512 & 0.013143 & 0.006492 & 0.003879 & 0.002729 \\
\hline \hline
\end{tabular}

Table 3 reports the average premium coefficients of the original tariff structures, at stationarity. The result shows that BMS based on these tariff structures are characterised by a significant lack of transparency, since all $C_{m}^{S}(\infty)$ are much less than one.

TABLE 3

AVERAGe PREMIUM COEFFICIENT

\begin{tabular}{ll}
\hline \hline Country & $C_{m}^{\boldsymbol{S}(\infty)}$ \\
\hline Kenya & 0.601557 \\
UK & 0.405212 \\
Brazil & 0.664135 \\
\hline \hline
\end{tabular}


Then under the transparency condition we determine the tariff structure for each system by using the risk class methodology, the original methodology and the one obtained by solving the constrained optimum problem (9). The average rating error related to the whole portfolio derives from the premium coefficients sequence. We use it as a measure to compare different tariff structures in terms of adequacy.

Results appear in Tables 4, 5 and 6, arranged by country:

TABLE 4

KENYAN PREMIUM COEFFICIENTS FOR DEFINED TARIFF STRUCTURES AND AVERAGE RATING ERRORS

\begin{tabular}{lcccccccc}
\hline \hline Kenya & $\boldsymbol{A} \boldsymbol{m}\left(\boldsymbol{c}_{\boldsymbol{i}}\right)$ & $\boldsymbol{c}_{\boldsymbol{1}}$ & $\boldsymbol{c}_{\boldsymbol{2}}$ & $\boldsymbol{c}_{3}$ & $\boldsymbol{c}_{\boldsymbol{4}}$ & $\boldsymbol{c}_{\boldsymbol{5}}$ & $\boldsymbol{c}_{\boldsymbol{6}}$ & $\boldsymbol{c}_{\boldsymbol{7}}$ \\
\hline Optimal & 0.009553 & 0.4962 & 1.0859 & 1.1856 & 1.3048 & 1.4496 & 1.6290 & 1.8569 \\
Original & 0.010246 & 0.6649 & 0.8312 & 0.9974 & 1.1636 & 1.3299 & 1.4961 & 1.6624 \\
Risk classes & 0.010846 & 0.7000 & 1.0852 & 1.1440 & 1.2095 & 1.2829 & 1.3659 & 1.4604 \\
\hline \hline
\end{tabular}

TABLE 5

BRITISH PREMIUM COEFFICIENTS FOR DEFINED TARIFF STRUCTURES AND AVERAGE RATING ERRORS

\begin{tabular}{lcccccccc}
\hline \hline$U \boldsymbol{U}$ & $\boldsymbol{A m}\left(\boldsymbol{c}_{\boldsymbol{i}}\right)$ & $\boldsymbol{c}_{\boldsymbol{I}}$ & $\boldsymbol{c}_{\boldsymbol{2}}$ & $\boldsymbol{c}_{\boldsymbol{3}}$ & $\boldsymbol{c}_{\boldsymbol{4}}$ & $\boldsymbol{c}_{5}$ & $\boldsymbol{c}_{\boldsymbol{6}}$ & $\boldsymbol{c}_{7}$ \\
\hline Optimal & 0.010179 & 0.6287 & 1.2645 & 1.3752 & 1.5048 & 2.1441 & 2.6716 & 3.3170 \\
Original & 0.013791 & 0.8144 & 0.9871 & 1.1105 & 1.3573 & 1.6041 & 1.8509 & 2.4678 \\
Risk classes & 0.013276 & 0.7726 & 1.1746 & 1.2383 & 1.3087 & 1.7050 & 1.9865 & 2.3578 \\
\hline \hline
\end{tabular}

TABLE 6

BRAZILIAN PREMIUM COEFFICIENTS FOR DEFINED TARIFF STRUCTURES AND AVERAGE RATING ERRORS

\begin{tabular}{lcccccccc}
\hline \hline Brazil & $\boldsymbol{A} \boldsymbol{m}\left(\boldsymbol{c}_{\boldsymbol{i}}\right)$ & $\boldsymbol{c}_{\boldsymbol{1}}$ & $\boldsymbol{c}_{\boldsymbol{2}}$ & $\boldsymbol{c}_{\boldsymbol{3}}$ & $\boldsymbol{c}_{\boldsymbol{4}}$ & $\boldsymbol{c}_{5}$ & $\boldsymbol{c}_{6}$ & $\boldsymbol{c}_{7}$ \\
\hline Optimal & 0.011594 & 0.8050 & 1.5524 & 2.2004 & 2.7920 & 3.3493 & 3.9015 & 4.4804 \\
Original & 0.044992 & 0.9787 & 1.0540 & 1.1293 & 1.2046 & 1.2799 & 1.3551 & 1.5057 \\
Risk classes & 0.016483 & 0.8778 & 1.3273 & 1.7515 & 2.1643 & 2.5654 & 2.9593 & 3.3526 \\
\hline \hline
\end{tabular}

The values displayed in Table 1 converge over time to those of the original tariff structure under transparency condition. In fact, this is due to the adjustments applied, year after year, to the premium coefficients. As a result, an increase in the number of malus classes will occur, the driver being clearly unaware of it.

On the other hand, using the tariff developed with a transparency condition, the insured will always be aware of the exact amount due.

Looking also at the adequacy of the system, we notice that all of the tariff structures analysed penalise claims more heavily than the original tariff. 
Then, if we use only the average rating error of the whole portfolio, we are unable to analyse the sensitivity of the system adequacy to different tariff structures. In order to develop a more specific analysis, we use the average rating error related to a non-personalised system in order to provide a measure of the adequacy improvement. The measure is the ratio of the observed average error using different premium coefficients, and the error arising from a non-personalised system. Results are reported in Table 7.

TABLE 7

AVERAGE RATING ERRORS AND RATIOS WITH NO-PERSONALISED BMS

\begin{tabular}{lcccccc}
\hline \hline \multirow{2}{*}{ Tariff } & \multicolumn{2}{c}{ Kenya } & \multicolumn{2}{c}{ UK } & \multicolumn{2}{c}{ Brazil } \\
\cline { 2 - 7 } & \multirow{2}{*}{$\boldsymbol{A m}\left(\boldsymbol{c}_{\boldsymbol{i}}\right)$} & \multicolumn{1}{c}{ ratio } & Am $\left(\boldsymbol{c}_{\boldsymbol{i}}\right)$ & ratio & Am( $\left.\boldsymbol{c}_{\boldsymbol{i}}\right)$ & \multicolumn{1}{c}{ ratio } \\
\hline Optimal & 0.009553 & $57.0 \%$ & 0.010179 & $35.0 \%$ & 0.011594 & $20.8 \%$ \\
Original & 0.010246 & $61.2 \%$ & 0.013791 & $47.4 \%$ & 0.044992 & $80.6 \%$ \\
Risk-classes & 0.010846 & $64.7 \%$ & 0.013276 & $45.6 \%$ & 0.016483 & $29.5 \%$ \\
Non-personalised & 0.016752 & $100.0 \%$ & 0.029092 & $100.0 \%$ & 0.055820 & $100.0 \%$ \\
\hline \hline
\end{tabular}

After liberalisation, Italian insurance companies presently selling automobile insurance can choose their own premium coefficients and also their transition rules. So we believe it interesting for our analysis to evaluate the impact of transition rules on the system adequacy in the three countries just considered. Assuming that the same premium scale is used in each country, under the transparency condition we obtain Table 8 .

TABLE 8

AVERAGE RATING ERRORS FOR DIFFERENT TARIFF STRUCTURES AND TRANSITION RULES

\begin{tabular}{lccc}
\hline \hline$T_{k} c_{i}^{S}$ & Kenya & UK & Brazil \\
\hline Kenya & 0.010246 & 0.010294 & 0.013001 \\
UK & 0.015424 & 0.013791 & 0.022738 \\
Brazil & 0.028906 & 0.025862 & 0.044992 \\
\hline \hline
\end{tabular}

\section{Conclusions}

The EU directives have enabled Italian motor insurance companies to change premium coefficients and transition rules. All tariffs created after the liberalisation, however, did not solve the problem arising from the lack of transparency. 
Our analysis suggest that, in respect of transparency and financial balance conditions, both premium coefficients sequence and transition rules are necessary tools in order to penalise drivers more heavily and to obtain a better portfolio of risks in terms of adequacy. The model developed here works on tariff structures and increases the adequacy of the system, at the same time guaranteeing transparency in the long run.

\section{REFERENCES}

LemAIRE, J. (1985) Automobile insurance: actuarial models. Kluwer-Nijhoff Publishing, Boston. LemaIRE, J. (1995) Bonus-Malus systems in automobile insurance. Kluwer-Academic Publishers, Boston.

Loimaranta, K. (1972) Some Asymptotic Properties of Bonus Systems. ASTIN Bulletin 6, 233245.

Fabio Baione, Susanna Levantesi and Massimiliano Menzietti

Dipartimento di Scienze Attuariali e Finanziarie,

Via Nomentana, 41

C.A.P. 00161 Roma, Italia

E-mail: fabio.baione@uniroma1.it

susanna.levantesi@uniroma1.it

massimiliano.menzietti@uniroma1.it 


\section{APPENDIX}

The following figures are the graphical representations of the density functions $u_{i}(\lambda),(i=1,2, \ldots, 7)$ for Kenya, United Kingdom and Brazil:

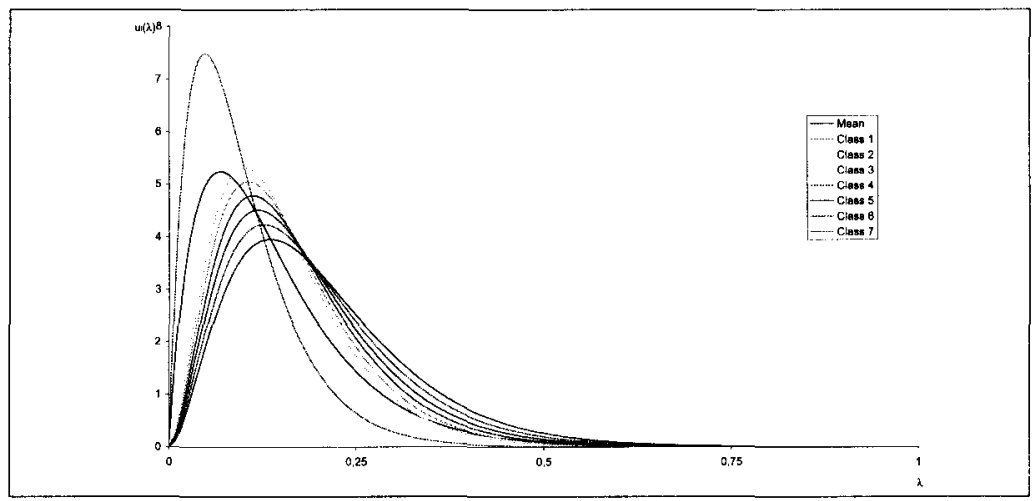

Figure 1. Kenyan density function

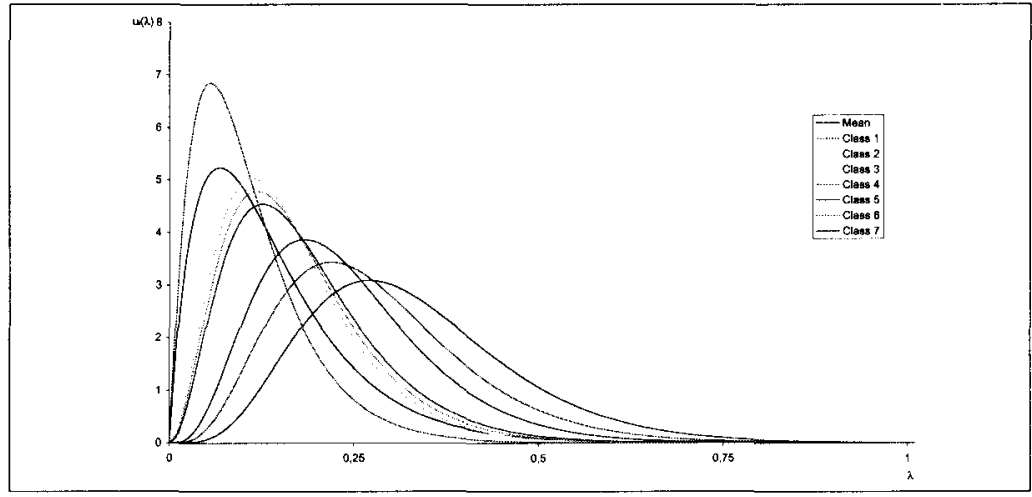

Figure 2. British density function

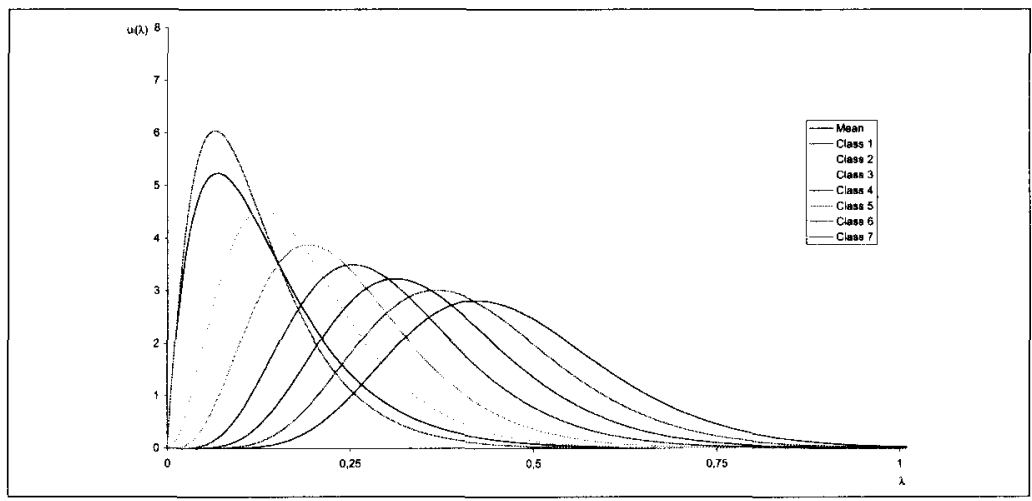

Figure 3. Brazilian density function 